\title{
On distribution functions with a limiting stable distribution function
}

\author{
By Harald Bergström
}

\section{Introduction ${ }^{1}$}

The general stable d.f.'s ${ }^{2}$ have been introduced by P. LÉvY ${ }^{3}$ who defined them implicitly by help of their characteristic functions and explicitly as limiting distribution functions. To every $\alpha, 0<\alpha \leq 2$ there belong stable distribution functions $G_{\alpha}(x)$ and these have the following property. ${ }^{4}$ If $*$ denotes the convolution and $\sigma_{1}, \sigma_{2}$ and $\sigma$ are positive numbers with

$$
\sigma_{1}^{\alpha}+\sigma_{2}^{\alpha}=\sigma^{\alpha}
$$

we have

$$
G_{\alpha}\left(\frac{x}{\sigma_{1}}\right) * G_{a}\left(\frac{x}{\sigma_{2}}\right)=G_{a}\left(\frac{x}{\sigma}\right) .
$$

In the following we shall only use the property (2) and the fact that $G_{a}(x)$ has derivatives of bounded variation of all orders. ${ }^{5}$

Let now $F(x)$ denote a d.f. and let $F^{* n}(x)$ denote the $n$-fold convolution of $F(x)$ with itself. W. DoEblin has given necessary and sufficient conditions which $F(x)$ must satisfy, if $F^{* n}\left(b_{n} x\right)$ shall converge to a stable d.f. $G_{\alpha}(x)$, $0<\alpha<2 .^{6}$ If $\alpha=2$ then $G_{\alpha}(x)$ is the normal d.f. and the conditions for convergence are then well known.

Our method can be used to get the conditions for convergence and we shall return to this problem later. Here we shall give estimations of the remainder term

1 Mr. Kai Lai Chung drew my attention to the general stable d.f.'s in a discussion which I had with him on the application of my methods.

d.f. - read distribution function(s).

3 P. Lévy (1), pp. 94-97, 198-204.

We call $\alpha$ the exponent of the stable d.f.

We omit the singular case, when $G_{\alpha}(x)$ is discontinuous.

${ }^{6}$ W. Dokblin (1), pn. 71-96. 
H. BERGSTRÖM, On disiribution functions

$$
F^{* n}(x)-G_{\alpha}^{* n}(x)
$$

when $F^{* n}\left(n^{\frac{1}{\alpha}} x\right)$ converges to $G_{a}^{* n}\left(n^{\frac{1}{\alpha}} x\right)=G_{\alpha}(x) .{ }^{1}$ Our main result is the following theorem. ${ }^{2}$

Theorem. Let $G(x)$ denote a stable d.f. of exponent $\alpha, 0<\alpha \leq 2$, and let $g(x)$ denote a non decreasing function such that there exist positive numbers $\lambda_{1}$ and $\lambda_{2}$ with $\lambda_{2} \leq\left[\lambda_{1}\right]+1$ for which

$$
\frac{1}{a}\left(\frac{x}{y}\right)^{\lambda_{1}} \leq\left(\frac{x}{y}\right)^{a} \frac{g(x)}{g(y)} \leq a\left(\frac{x}{y}\right)^{\lambda_{2}}
$$

with a constant a when $x>y>0 .^{3}$ Further suppose that $F(x)$ is a d.f. which satisfies the following conditions

10

$$
\int_{-\infty}^{\infty}|x|^{\alpha} g(|x|)|d[F(x)-G(x)]|<\infty
$$

$2^{\circ}$

$$
\int_{-\infty}^{\infty} x^{\nu} d\left[F^{\prime}(x)-G(x)\right]=0
$$

for $\nu=0,1, \ldots, v_{0} . \quad$ Putting

$$
\delta(n)=\left\{\begin{array}{lll}
g^{-1}\left(n^{\frac{1}{a}}\right) & \text { if } & \nu_{0} \geq\left[\lambda_{1}\right] \\
n^{-\frac{v_{0}+1-a}{a}} & \text { if } & \nu_{0}<\left[\lambda_{1}\right]
\end{array}\right.
$$

we then have for fixed $v$ and large $n$

$$
\left(\begin{array}{l}
n \\
v
\end{array}\right) G^{* n-v}(x) *[F(x)-G(x)]^{* v}= \begin{cases}0\left[\delta^{\nu}(n)\right] & \text { always }, \\
0\left[\delta^{v}(n)\right], & \text { if } \quad v_{0} \geq\left[\lambda_{1}\right]\end{cases}
$$

Assuming that

$$
\lambda_{2}<\left[\lambda_{1}\right]+1 \text {. }
$$

$3^{\circ}$

$$
\sum_{n=1}^{\infty} n^{-1} g^{-(s+1)}\left(n^{\frac{1}{a}}\right)<\infty
$$

1 In the general case $F^{* n}\left(b_{n} x\right)$ converges to $G_{\alpha}(x)$ when $b_{n}$ is some suitable possibly more complicated increasing function of $n$. Compare Dosblin loc. cit.

${ }^{2}$ In the following we write $G(x)$ instead of $G_{a}(x)$.

3 We can for instance consider $g(x)=x^{\varrho}$ with some exponent $\varrho>0$ or $g(x)=\log x$. If $\lim _{x \rightarrow \infty} \frac{g(c x)}{g(x)}=\gamma(c) \neq 0$ for some $c$ in the interval $0<c<1$ we may choose $\lambda_{1}-\alpha$ and $\lambda_{2}-\alpha$ arbitrarily close to $\frac{\log \gamma(c)}{\log c}$ and then $g(x)$ has the mentioned property. 
we have the asymptotic expansion

$$
F^{* n}(x)=\sum_{v=0}^{s}\left(\begin{array}{l}
n \\
v
\end{array}\right) G^{* n-\nu}(x) *[F(x)-G(x)]^{* v}+r_{n}^{(s+1)}
$$

with

$$
r_{n}^{(s+1)}= \begin{cases}0\left[\delta^{s+1}(n)\right]+0\left(n^{-\frac{1}{\alpha}}\right), & \text { always, } \\ 0\left[\delta^{s+1}(n)\right]+0\left(n^{-\frac{1}{\alpha}}\right), & \text { if } \quad v_{0} \geq\left[\lambda_{1}\right], \\ & \lambda_{2}<\left[\lambda_{1}\right]+1 .\end{cases}
$$

If furthermore the condition ${ }^{1}$

$4^{\circ}$

$$
\lim _{p \rightarrow \infty} V\left\{F^{* p}(x) *[F(x)-G(x)]\right\}=0
$$

is satisfied then $0\left(n^{-\frac{1}{\alpha}}\right)$ may be omitted in (iii).

Remark I. The estimations of $r_{n}^{(s+1)}$ in (iii) can be given such that they are independent on other quantities than $s, \alpha, \lambda_{1}, \lambda_{2}, \nu_{0}$ and $n$. If $4^{\circ}$ holds and $0\left(n^{-\frac{1}{\alpha}}\right)$ is omitted the estimation is more dependent on $F(x)$.

Remark II. Independently on the condition $3^{0}$ the expansion (ii) is asymptotic with

$$
r_{n}^{(s+1)}= \begin{cases}0\left[\delta^{s+1}(n)\right]+0\left[\delta \frac{1}{\lambda_{2}}(n)\right]+0\left(n^{-\frac{1}{\alpha}}\right) & \text { always } \\ 0\left[\delta^{s+1}(n)\right]+0\left[\delta_{\frac{\lambda_{2}}{\lambda_{2}}}(n)\right]+0\left(n^{-\frac{1}{\alpha}}\right), & \text { if } \quad v_{0} \geq\left[\lambda_{1}\right], \\ & \lambda_{2}<\left[\lambda_{1}\right]+1 .\end{cases}
$$

Remark III. If we change the assumptions $1^{\circ}$ and $2^{\circ}$ to

$10 \mathrm{a}$

$$
\int_{-\infty}^{\infty}|x|^{\alpha-1} g(|x|)|F(x)-G(x)| d x<\infty
$$

and

$20 \mathrm{a}$

$$
\int_{-\infty}^{\infty} x^{y}[F(x)-G(x)] d x=0
$$

for $\nu=0,1, \ldots, \nu_{0}-1$, the theorem still holds.

Remark IV. The theorem can easily be generalized to the case of multidimensional d.f. ${ }^{2}$

$1 V\{\}$ denotes the total variation.

2 Compare H. Bergström (2). 


\section{H. BERGSTRöM, On distribution functions}

If we can chose $g(x)=x^{r}$ with some $r>0$, it follows from (ii) and (iii) that $F^{* n}(x)$ cannot have jumps of larger order of magnitude than $n^{-\frac{1}{a}}$. In the case $\alpha=2$ we know that $F^{* n}(x)$ has jumps of the order $n^{-\frac{1}{a}}$, if for instance $F(x)$ is the Bernoullian distribution function.

We shall prove the theorem by the same method that we have used for the proof of the corresponding special theorem for the normal d.f. ${ }^{1}$ In fact we have only to change that proof at some points in order to get the proof of the general theorem. However, we shall give the complete proof here.

We start by proving a lemma.

Lemma. Let $g(x), F(x), G(x), \lambda_{1}, \lambda_{2}$ and $\propto$ be defined as in the theorem, and suppose that the condition $1^{\circ}$ is satisfied. Then the moments

$$
\int_{-\infty}^{\infty} x^{v} d[F(x)-G(x)]=\beta^{(v)}
$$

exist for $\nu=0, \ldots,\left[\lambda_{1}\right]$, and if $\psi(x)$ is a function with bounded continuous derivatives of all orders $\leq\left[\lambda_{1}\right]+1$ for all $x$, we have for $p>0$

$$
\begin{aligned}
\psi\left(\frac{x}{p}\right) *[F(x)- & G(x)]=\sum_{\nu=0}^{\left[\lambda_{1}\right]} \frac{(-1)^{\nu} \beta^{(\nu)}}{\nu ! p^{\nu}} \psi^{(v)}\left(\frac{x}{p}\right)+ \\
& +0\left[p^{-\alpha} g^{-1}(p)\right]\left\{\underset{x}{\operatorname{Max}}\left|\psi^{\left.\left(\lambda_{1}\right]\right)}\left(\frac{x}{p}\right)\right|+\underset{x}{\operatorname{Max}}\left|\psi^{\left(\left[\lambda_{1}\right]+1\right)}\left(\frac{x}{p}\right)\right|\right\} .
\end{aligned}
$$

Here 0 may be changed against o if $\lambda_{2}<\left[\lambda_{1}\right]+1$.

Proof: We put $h(x)=x^{\alpha} g(x),\left[\lambda_{1}\right]=\lambda$. Expanding $\psi\left(\frac{x-t}{p}\right)$ by Taylor's formula we get

$$
\psi\left(\frac{x-t}{p}\right)=\sum_{\nu=0}^{\lambda} \frac{(-1)^{\nu} t^{\nu}}{\nu ! p^{\nu}} \psi^{(p)}\left(\frac{x}{p}\right)+g_{\lambda}(x, t),
$$

where the remainder term may be written in either of the forms

$$
g_{\lambda}(x, t)=\frac{(-1)^{\lambda}}{\lambda !}\left(\frac{t}{p}\right)^{\lambda}\left[\psi^{(\lambda)}\left(\frac{x-\Theta t}{p}\right)-\psi^{(\lambda)}\left(\frac{x}{p}\right)\right], \quad 0<\Theta<1
$$

or

$$
g_{\lambda}(x, t)=\frac{(-1)^{\lambda+1}}{(\lambda+1) !}\left(\frac{t}{p}\right)^{\lambda+1} \psi^{(\lambda+1)}\left(\frac{x-\Theta_{1} t}{p}\right), \quad 0<\Theta_{1}<1 .
$$

Owing to $1^{0}$ the moments $\beta^{(\nu)}$ exists for $\nu=0, \ldots, \lambda$. Applying (4 a) and (4 b) we then get

\footnotetext{
1 H. Bergstróm (2), p. 5.
} 


$$
\begin{aligned}
\psi\left(\frac{x}{p}\right) *[F(x)-G(x)]=\int_{-\infty}^{\infty} \psi\left(\frac{x-t}{p}\right) d[F(t]-G(t)]= \\
=\sum_{\nu=0}^{1} \frac{(-1)^{\nu} \beta^{(\nu)}}{\nu ! p^{\nu}} \psi^{(\nu)}\left(\frac{x}{p}\right)+\int_{-\infty}^{\infty} g_{\lambda}(x, t) d[F(t)-G(t)] .
\end{aligned}
$$

Using (4 a) for $|t|>b$ and (4 b) for $|t| \leq b$ and observing that, owing to the made assumption about $g(x)$,

$$
\begin{gathered}
\left|t^{\lambda}\right| \leq a \frac{b^{\lambda}}{h(b)} h(|t|) \text { for }|t| \geq b, \\
\left|t^{\lambda+1}\right| \leq a \frac{b^{\lambda+1}}{h(b)} h(|t|) \text { for }|t| \leq b,
\end{gathered}
$$

we get

$$
\begin{aligned}
\left|\int_{-\infty}^{\infty} g_{\lambda}(x, t) d[F(t)-G(t)]\right| \leq \\
\quad \leq \frac{2 b^{\lambda} a}{\lambda ! h(b) p^{\lambda}} \operatorname{Max}_{x}\left|\psi^{(\lambda)}\left(\frac{x}{p}\right)\right| \int_{|t|>b} h(|t|)|d[F(t)-G(t)]|+ \\
\quad+\frac{b^{\lambda+1} a}{(\lambda+1) ! h(b) p^{\lambda+1}} \operatorname{Max}_{x}\left|\psi^{(\lambda+1)}\left(\frac{x}{p}\right)\right| \int_{|t| \leq b} h(|t|)|d[F(t)-G(t)]| .
\end{aligned}
$$

Putting

$$
r(b)=\int_{|t| \geq 0} h(|t|)|d[F(t)-G(t)]|
$$

we now have to consider the quantities

$$
\frac{b^{\lambda} r(b)}{h(b) p^{\lambda}} \text { and } \frac{b^{\lambda+1}}{h(b) p^{\lambda+1}}
$$

Here $r(b)$ is bounded for $b=0$ and nonincreasing for $b>0$ with $\lim _{b \rightarrow \infty} r(b)=0$. If we choose $b=p$ the quantities (5) are both $0\left[h^{-1}(p)\right]$. Therefore (i) holds. But for $b<p$ we have more over

(6)

$$
\left\{\begin{array}{l}
\frac{b^{\lambda} r(b)}{h(b) p^{\lambda}}=\left(\frac{b}{p}\right)^{\lambda} \frac{h(p)}{h(b)} \frac{r(b)}{h(p)} \leq a \frac{r(b)}{h(p)}\left(\frac{p}{b}\right)^{\lambda_{2}-\lambda} \\
\frac{b^{\lambda+1}}{h(b) p^{\lambda+1}}=\left(\frac{b}{p}\right)^{\lambda+1} \frac{h(p)}{h(b)} \frac{1}{h(p)} \leq \frac{a}{h(p)}\left(\frac{b}{p}\right)^{1+\lambda-\lambda_{2}}:
\end{array}\right.
$$




\section{H. BERGSTRÖM, On distribution functions}

If $\lambda_{2}<1+\lambda$ it is obviously possible to let $b=b(p)<p$ tend to infinity in such a way that the right sides of $(6)$ are $o\left[\frac{1}{h(p)}\right]$. Thus the lemma is proved.

We are now going to prove the theorem. Then it is sufficient to consider the case $v_{0} \geq\left[\lambda_{1}\right]$, for if $v_{0}<\left[\lambda_{1}\right]$ we may choose $g(x)=x^{y_{0}+1-a}$.

We get the relation (i) of the theorem in the case $v=1, v_{0} \geq \lambda=\left[\lambda_{1}\right]$ if we apply the lemma with

$$
\psi\left(\frac{x}{p}\right)=G^{* n-1}(x)=G\left(\frac{x}{p}\right), \quad p=(n-1)^{\frac{1}{a}} .
$$

Generally (i) of the theorem may be proved by help of induction. Putting

$$
\psi\left(\frac{x}{p}\right)=G\left(\frac{x}{p}\right) *[F(x)-G(x)]^{*-1}, \quad p=(n-\nu+1)^{\frac{1}{a}}
$$

we get (i) of the lemma. Here is

$$
\psi^{(\mu)}\left(\frac{x}{p}\right)=G^{(\mu)}\left(\frac{x}{p}\right) *[F(x)-G(x)]^{* v-1}
$$

and we can apply the lemma again putting now

$$
\psi\left(\frac{x}{p}\right)=G^{(\mu)}\left(\frac{x}{p}\right) *[F(x)-G(x)]^{* v-2}
$$

for $\mu=\lambda$ and $\mu=\lambda+1$. In this way we prove (i) of the theorem by help of induction.

In order to prove (iii) we shall also use induction. We assume that the inequality

$$
\left|r_{\varrho}^{(s+1)}\right|<C \delta_{1}^{s+1}(\varrho)
$$

holds for $\varrho<n$ with

$$
\delta_{1}(\varrho)= \begin{cases}\operatorname{Max}\left[g^{-(s+1)}\left(\varrho^{\frac{1}{\alpha}}\right), \varrho^{-\frac{1}{\alpha}}\right], & \text { if } \quad v_{0} \geq \lambda \\ \operatorname{Max}\left[\varrho^{-(s+1) \frac{\left(v_{0}+1-a\right)}{\alpha}}, \varrho^{-\frac{1}{\alpha}}\right], \quad \text { if } \quad v_{0}<\lambda\end{cases}
$$

and a constant $C$ and then we prove that

$$
r_{n}^{(s+1)}<C(n) \delta_{1}^{s+1}(n)
$$

where $C(n)<C$ if $n$ is larger than some constant $n_{0}$ and also

and

$$
C(n)=o(n), \quad \text { if } \quad \nu_{0} \geq\left[\lambda_{1}\right], \lambda_{2}<\left[\lambda_{1}\right]+1
$$

$$
n^{-\frac{1}{\alpha}}=0\left[\delta_{1}^{s+1}(n)\right] .
$$


Obviously (7) holds with a sufficiently large constant $C$ for $n \leq n_{0}$. Thus the theorem follows by induction.

In order to prove (8), we consider

$$
r_{n}^{(s+1)}(x) * \phi\left(\frac{x}{p_{1}}\right)
$$

where $\phi(x)$ is the normal d.f. with the mean value 0 and the dispersion 1 , and give an estimation in the form

$$
\left|r_{n}^{(s+1)}(x) * \phi\left(\frac{x}{p_{1}}\right)\right|<M\left(n, p_{1}\right),
$$

where $M\left(n, p_{1}\right)$ depends on $n, p_{1}$ and $C$. Then applying a lemma for Weierstrass singular integral ${ }^{\mathbf{1}}$, and observing that

$$
\frac{d}{d x} G^{* n}(x)=n^{-\frac{1}{\alpha}} G^{\prime}\left(n^{-\frac{1}{\alpha}} x\right)
$$

we obtain

$$
\left|r_{n}^{s+1}\right| \leq \operatorname{Max}\left[k M\left(n, p_{1}\right), \beta p_{1} n^{-\frac{1}{a}}\right]
$$

with constants $k$ and $\beta$. By suitable choice of $p_{1}$ it can then be proved that (8) holds.

For abbreviation we put

$$
\left(\begin{array}{l}
n \\
v
\end{array}\right) G^{* n-\nu}(x) *[F(x)-G(x)]^{* v}=\Delta_{n}^{(v)} .
$$

Owing to an identical expansion we have ${ }^{2}$

$$
r_{n}^{(s+1)}=\sum_{\mu=s+1}^{n}\left(\begin{array}{c}
\mu-1 \\
s
\end{array}\right) F^{* n-\mu} *(F-G)^{* s+1} * G^{* \mu-s-1},
$$

i.e.

$$
r_{n}^{(s+1)}=\sum_{\mu=s+1}^{n} F^{* n-\mu} * \Delta_{\mu-1}^{(s)} *(F-G)
$$

and

$$
F^{* n}=\sum_{\nu=0}^{s} \Delta_{n}^{(\nu)}+r_{n}^{(s+1)}
$$

Let $m_{1}=\left[\frac{n}{2}\right], n>n_{0}$ where $n_{0}$ is a suitable fixed integer. Observing that then owing to the property of $g(x)$

$$
\frac{\delta\left(m_{1}\right)}{\delta(n)}>c_{0}
$$

1 H. Bergström (1), p. 143.

2 H. Bergström (2), p. 2. 


\section{H. BERGSTRÖM, On distribution functions}

with a constant $c_{0}$, we get in the same way as we have found (i) of the theorem

$$
\begin{aligned}
\left|\sum_{\mu=m_{1}+1}^{n} F^{* n-\mu} * \Delta_{\mu-1}^{(s)} *(F-G)\right| \leq \operatorname{Max}_{x} \sum_{\mu=m_{1}+1}^{n}\left|\Delta_{\mu-1}^{(s)} *(F-G)\right|< \\
<a_{0}(n) \delta^{s+1}(n)
\end{aligned}
$$

where $a_{0}(n)$ is smaller than some constant $c_{1}$ for all $n$ and tends to zero for large $n$ if $\nu_{0} \geq\left[\lambda_{1}\right], \lambda_{2}<\left[\lambda_{1}\right]+1$. In order to estimate the members in the right side of (11) for $\mu \leq m_{1}$, we express $F^{* n}$ by (12) changing $n$ to $n-\mu$. Then we get

$$
\begin{aligned}
\sum_{\mu=s+1}^{m_{1}} F^{* n-\mu} * \Delta_{\mu-1}^{(s)} *(F-G)=\sum_{\mu=s+1}^{m_{1}} \sum_{\nu=0}^{s} \Delta_{n-\mu}^{(v)} * \Delta_{\mu-1}^{(s)} *(F-G)+ & \\
& +\sum_{\mu=s+1}^{m_{1}} r_{n-\mu}^{(s+1)} * \Delta_{\mu-1}^{(s)} *(F-G) .
\end{aligned}
$$

Analogous to (13) we find for $n>n_{0}$

$$
\left|\sum_{\mu=s+1}^{m_{1}} \sum_{\nu=0}^{s} \Delta_{n-\mu}^{(v)} * \Delta_{\mu-1}^{(s)} *(F-G)\right|<a_{1}(n) \delta^{s+1}(n)
$$

where $a_{1}(n)$ is defined in the same way as $a_{0}(n)$.

Further we have with an integer $m_{2} \leq m_{1}$.

$$
\begin{aligned}
& \left|\sum_{\mu=s+1}^{m_{1}} r_{n-\mu}^{(s+1)} * \Delta_{\mu-1}^{(s)} *(F-G) * \phi\left(\frac{x}{p_{1}}\right)\right| \leq \\
& \quad \leq \sum_{\mu=m_{2}+1}^{m_{1}}\left(\begin{array}{c}
\mu-1 \\
s
\end{array}\right) \underset{x}{\operatorname{Max}}\left|r_{n-\mu}^{(s+1)} * G^{* \mu-s-1} *(F-G)^{* s+1}\right|+ \\
& \quad+\sum_{\mu=s+1}^{m_{2}}\left(\begin{array}{c}
\mu-1 \\
s
\end{array}\right) \underset{x}{\operatorname{Max}_{x}\left|r_{n-\mu}^{(s+1)} * \phi\left(\frac{x}{p_{1}}\right) *(F-G)^{* s+1}\right| .}
\end{aligned}
$$

In the members of the first sum of the right side of (16) we apply the lemma with

$$
\psi\left(\frac{x}{p}\right)=r_{n-\mu}^{(s+1)} * G\left(\frac{x}{p}\right), \quad p=(\mu-s-1)^{\frac{1}{a}}
$$

and observe that for any positive integer $y$

$$
\left|\psi^{(v)}\left(\frac{x}{p}\right)\right|=\left|r_{n-\mu}^{(s+1)} * G^{(\nu)}\left(\frac{x}{p}\right)\right| \leq \operatorname{Max}_{x}\left|r_{n-\mu}^{(s+1)}\right| \int_{-\infty}^{\infty}\left|G^{(v+1)}(x)\right| d x^{2}
$$

1 The "constants" $c$ in the following depend only on the quantities $a, s, \lambda_{1}, \lambda_{2}, \alpha$ and $v_{0}$ defined in the theorem.

${ }_{2}$ All derivatives of $G(x)$ are of bounded variation, what for instance easily may be obtained from Bergsthöm (3). 
Assuming that (7) holds for $\varrho<n$ and observing that

$$
\delta_{1}(n-\mu)<c_{2} \delta_{1}(n)
$$

with a constant $c_{2}$ for $\mu \leq m_{1}$ we get

$$
\left|r_{n-\mu}^{(s+1)}\right|<C c_{3} \delta_{1}^{(s+1)}(n)
$$

with a constant $c_{3}$ and thus according to the lemma

$$
\begin{aligned}
\sum_{\mu=m_{2}+1}^{m_{1}}\left(\begin{array}{c}
\mu-1 \\
s
\end{array}\right) \underset{x}{\operatorname{Max}_{x} \mid r_{n-\mu}^{(s+1)} *} G^{* \mu-s-1} *(F-G)^{* s+1} \mid< \\
\quad<C \delta_{1}^{s+1}(n) a_{2}\left(m_{2}\right) \sum_{\mu=m_{2}+1}^{m_{1}} \frac{1}{\mu-1} \delta^{s+1}(\mu-1),
\end{aligned}
$$

where $a_{2}\left(m_{2}\right)$ is defined in the same way as $a_{0}(n)$. In the members of the second sum of the right side of (16) we apply the lemma with

$$
\psi\left(\frac{x}{p}\right)=r_{n-\mu}^{(s+1)} * \phi\left(\frac{x}{p_{1}}\right), \quad p=p_{1} .
$$

Then we get in the same way as we have obtained (17)

$$
\begin{aligned}
\sum_{\mu=s+1}^{m_{2}}\left(\begin{array}{c}
\mu-1 \\
s
\end{array}\right) \operatorname{Max}_{x} \mid r_{n-\mu}^{(s+1)} * \phi & \left(\frac{x}{p_{1}}\right) *(F-G)^{* s+1} \mid \\
& <C \delta_{1}^{s+1}(n) a_{3}\left(p_{1}\right)\left(\begin{array}{c}
m_{2} \\
s+1
\end{array}\right) p_{1}^{-\alpha(s+1)} \delta^{s+1}\left(p_{1}^{\alpha}\right)
\end{aligned}
$$

where $a_{3}\left(p_{1}\right)$ is defined in the same way as $a_{0}(n)$. Combining (11), (13), (15), (17) and (18), we find that we can choose $M\left(n, p_{1}\right)$ in (9) equal to

$$
\begin{aligned}
& M\left(n, p_{1}\right)=\delta^{s+1}(n)\left[a_{0}(n)+a_{1}(n)\right]+ \\
& \quad+\delta_{1}^{s+1}(n) C\left[a_{2}\left(m_{2}\right) \sum_{\mu=m_{2}+1}^{m_{1}} \frac{1}{\mu-1} \delta^{s+1}(\mu-1)+\right. \\
& \left.\quad+a_{3}\left(p_{1}\right)\left(\begin{array}{c}
m_{2} \\
s+1
\end{array}\right) p_{1}^{-\alpha(s+1)} \delta^{s+1}\left(p_{1}^{\alpha}\right)\right]
\end{aligned}
$$

Owing to (10) we have then to show that

$$
\underset{x}{\operatorname{Max}}\left[k M\left(n, p_{1}\right), \beta p_{1} n^{-\frac{1}{a}}\right]<C \delta_{1}^{s+1}(n) .
$$

Now the sum in the right side of (19) is the partial sum of a convergent series. (If $\delta(n)=n^{-\frac{v_{0}+1-\alpha}{a}}$ we may assume $\nu_{0}+1-\alpha>0$ for otherwise there is nothing to prove.) 


\section{H. BERGSTRÖM, On distribution functions}

Therefore we may choose $m_{2}$ so large that

$$
k a_{2}\left(m_{2}\right) \sum_{\mu=m_{2}+1}^{m_{1}} \frac{1}{\mu-1} \delta^{s+1}(\mu-1)<\frac{1}{3}
$$

where $k$ is given in (10). Having thus determined $m_{2}$, we may choose $p_{1}$ so large that

$$
k a_{3}\left(p_{1}\right)\left(\begin{array}{c}
m_{2} \\
s+1
\end{array}\right) p_{1}^{-\alpha(s+1)} \delta^{s+1}\left(p_{1}^{\alpha}\right)<\frac{1}{3} .
$$

If further $C$ is so large that

$$
k\left[a_{0}(n)+a_{1}(n)\right]<\frac{C}{3}
$$

we have

$$
k M\left(n, p_{1}\right)<C \delta^{s+1}(n)
$$

If $C$ also is so large that

$$
\beta p_{1}<C
$$

where $\beta$ is given in (10) it follows from (10) that (8) holds with $C(n)=C$, if (7) is satisfied for $\varrho<n$ and $n$ is larger than some value $n_{0}$. But (8) holds with a sufficiently large constant $C$ for $n \leq n_{0}$. Thus (2) holds with $C(n)=C$ for all $n$.

In order to prove the stronger inequality when $v_{0} \geq\left[\lambda_{1}\right], \lambda_{2}<\left[\lambda_{1}\right]+1$, we have only consider the case

$$
n^{-\frac{1}{a}}=0\left[\delta^{s+1}(n)\right], \quad \nu_{0} \geq\left[\lambda_{1}\right]
$$

Then we know by the just proved theorem that (8) holds with a constant $C$ and we get (19) with this known constant. It is then possible to let $p_{1}$ tend to infinity in such a way that

$$
\beta p_{1} n^{-\frac{1}{\alpha}}=0\left[\delta^{s+1}(n)\right]
$$

and to let $m_{2}$ tend to infinity in such a way that

and then is also

$$
\left(\begin{array}{c}
m_{2} \\
s+1
\end{array}\right) p_{1}^{-a(s+1)} \delta^{(s+1)}\left(p_{1}^{a}\right)=o(1)
$$

$$
\sum_{\mu=m_{2}+1}^{m_{1}} \frac{1}{\mu-1} \delta^{\mu-1}(\mu-1)=0(1)
$$

when $n$ tends to infinity. Therefore (iii) holds.

Let now the condition $4^{\circ}$ be satisfied. Then it follows from a general theorem ${ }^{1}$ that $0\left(n^{-\frac{1}{a}}\right)$ may be omitted.

1 H. Bergaström (2), p. 4. 
At last we have to prove the statement in the remarks of our theorem. The contents of the remarks I and III are immediately drawn from the proof given above. In order to prove the remark II, we only need to consider the case $\delta(n)=g^{-1}\left(n^{\bar{\alpha}}\right), \lambda_{1}=\alpha$. If we put

$$
\delta_{2}^{s+1}(n)=\operatorname{Max}\left[\delta^{s+1}(n), \delta^{\frac{1}{\lambda_{2}}}(n), n^{-\frac{1}{\alpha}}\right]
$$

and assume that (7) holds with $\delta_{2}$ instead of $\delta_{1}$ we obviously obtain (19) with $\delta_{2}(n)$ instead of $\delta_{1}(n)$. Choosing $m_{1}=m_{2}$, putting $p_{1}=n^{\frac{1}{\alpha}} q$ and observing that for $0<q<1$

$$
\frac{\delta\left(p_{1}^{a}\right)}{\delta(n)}=\frac{g\left(n^{\frac{1}{\alpha}}\right)}{g\left(n^{\frac{1}{\alpha}} q\right)}<a q^{-\left(\lambda_{2}-\alpha\right)}
$$

we may write (19) in the form

$$
M\left(n, p_{1}\right)<\delta^{s+1}(n)\left[a_{0}(n)+a_{1}(n)\right]+C a_{4}\left(n^{\frac{1}{a}} q\right) q^{-\lambda_{2}(s+1)} \delta_{2}^{s+1}(n) \delta^{s+1}(n) .
$$

Further (10) may be written

$$
\left|r_{n}^{(s+1)}\right| \leq \operatorname{Max}\left[k M\left(n, p_{1}\right), \beta q\right] .
$$

Now we choose

$$
g=C^{\frac{1}{\lambda_{2}(s+1)+1}} \delta_{2}^{\frac{1}{\lambda_{2}}}(n) .
$$

If then $C$ is so large that

$$
\begin{gathered}
k\left[a_{0}(n)+a_{1}(n)\right]<\frac{C}{2}, \\
k a_{4}(\stackrel{1}{\alpha} q) C^{-\frac{\lambda_{2}(s+1)}{\lambda_{2}(s+1)+1}}<\frac{1}{2}
\end{gathered}
$$

and

$$
\beta C^{-\frac{\lambda_{2}(s+1)}{\lambda_{2}(s+1)+1}}<1
$$

for $n>n_{0}$ and $n_{0}$ is so large that $q<1$ for $n>n_{0}$, it follows from (22) that

$$
r_{n}^{(s+1)}<C \delta_{2}^{s+1}(n)
$$

Since this inequality obviously holds for $n \leq n_{0}$ if $C$ is sufficiently large it then holds for all $n$ with suitable $C$. (If $\delta_{2}(n)$ doesn't tend to zero when $n$ tends to infinity it isn't perhaps possible to get $q<1$ but then (23) is trivial.)

In order to prove the stronger inequality when $\nu_{0} \geq\left[\lambda_{1}\right], \lambda_{2}<\left[\lambda_{1}\right]+1$, we now start from the true inequality (23) and then get (21). We have only to consider the case

$$
n^{-\frac{1}{a}}=o\left[\delta^{s+1}(n)\right]+o\left[\delta_{2}^{\overline{\lambda_{2}}}(n)\right]
$$




\section{H. BERGSTRöM, On distribution functions}

and then it is possible to choose

$$
q=0\left[\frac{1}{\delta_{2}^{\lambda_{2}}}(n)\right]
$$

in such a way that $n^{\bar{\alpha}} q \rightarrow \infty$ and

$$
a_{4}\left(n^{\frac{1}{\alpha}} q\right) q^{-\lambda_{2}(s+1)} \delta_{2}^{s+1}(n)=0(1) .
$$

Then the right side of $(21)$ is $o\left[\delta^{s+1}(n)\right]+o\left[\delta^{\frac{1}{\lambda_{2}}}(n)\right]$.

\section{LITERAT UR E}

H. Bergström (1): On the Central Limit Theorem. Skand. Aktuarietidskr. (1944), p. 139-152. (2): On Asymptotic Expansions of Probability Functions. Skand. Aktuarietidskr. (1951), pp. 1-33.

(3): On some expansions of stable distribution functions. Arkiv för Matematik, Band 2, No. $18,1952$.

W. Doeblin (1): Sur l'ensemble de puissance d'une loi de probabilité. Studia Math., vol. 9 (1941), pp. 71-96.

P. LÉvy (1): Théorie de l'addition des variables aléatoires. Gauthier-Willars (1937). 